\title{
A comparison of Miller and Otto cycle natural gas engines for small scale CHP applications.
}

\author{
R. Mikalsen *, Y.D. Wang, A.P. Roskilly \\ Sir Joseph Swan Institute for Energy Research, Newcastle University, Newcastle upon Tyne, NE1 7RU, United Kingdom.
}

\begin{abstract}
This paper presents an investigation into the feasibility and potential advantages of a small scale Miller cycle natural gas engine for applications such as domestic combined heat and power systems. The Miller cycle engine is compared to a standard Otto cycle engine using cycle analyses and multidimensional simulation, and basic engine design implications are discussed. It is found that the Miller cycle engine has a potential for improved fuel efficiency, but at the cost of a reduced power to weight ratio. A fuel efficiency advantage of $5 \rightarrow 10$ per cent compared to a standard Otto cycle engine appears possible, however it is stated that further investigations, in particular into the topic of engine friction, are required in order to validate the findings.
\end{abstract}

Key words: Miller cycle, natural gas engine, CHP, CFD.

\section{Introduction}

Domestic energy use, i.e. heating and electric power consumption, is the largest contributor to the average UK citizen's $\mathrm{CO}_{2}$ footprint [1], as the majority of this energy is generated using fossil fuels. As evidence of the environmental impacts of carbon dioxide emissions from fossil fuels becomes clearer and fuel costs increase, the interest in small scale, domestic combined heat and power (CHP) systems has increased. By utilising a part of the fuel energy to produce electricity locally, where the waste energy (typically two thirds of the fuel heat energy) is needed for heating, electric power consumption from large, fossil fuel based power plants, in which most of the excess heat energy is lost, can be reduced.

\footnotetext{
‡ This is a preprint version. This paper was published as: Applied Energy, Volume 86, Issue 6, June 2009, Pages 922927.

* Corresponding author. Tel. +44 1912464935

Email address: rikard@mikalsen.eu (R. Mikalsen).
}

1.1. Combined heat and power systems for small scale applications

The yearly energy consumption for a typical UK household consists of around $15 \rightarrow 25$ per cent electric energy and $75 \rightarrow 85$ per cent energy for heating. Although these numbers clearly have seasonal variations, this scales well with the efficiency one would expect from a combustion engine and electric generator set, which typically lies in the range $20 \rightarrow 35$ per cent, depending on size and application. Effective methods of coupling small CHP units to the electric grid exist, in order to account for variations in the supply from the unit and household demand.

Figure 1 shows two possible configurations for a small natural gas engine in domestic applications. Figure 1a shows a standard combined heat and power system, in which the mechanical output from the engine drives an electric generator. The electric power can be supplied to the grid, thereby reducing the household's electricity costs, and the exhaust heat from the engine is utilised for heating 


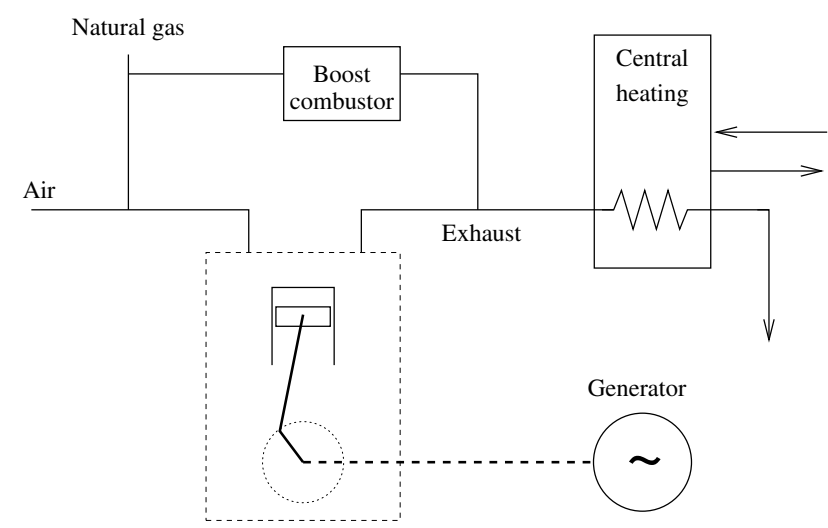

(a) Combined heat and electric power system.

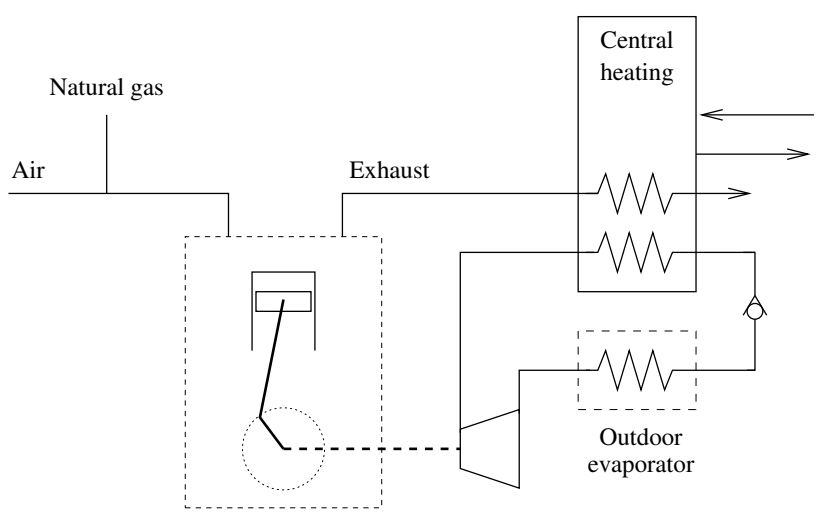

(b) Natural gas engine and heat pump system.

Fig. 1. Possible configurations for a natural gas engine in domestic applications.

purposes. Figure $1 \mathrm{~b}$ shows an alternative configuration of a heating-only system based on a natural gas engine driving a heat pump. Depending on the configuration, modern domestic heat pumps typically achieve a coefficient of performance (COP) of $3 \rightarrow 5$, i.e. for one unit of input energy $3 \rightarrow 5$ units of heat can be supplied. With an engine efficiency of $25 \%$ and a heat pump COP of 3 (a conservative estimate), a reduction in fuel use for heating in the order of $30 \%$ is possible. As an alternative the heat pump could be used to provide cooling, which could be an attractive solution in applications such as office buildings with rooms for computing equipment or convenience stores which require cooled storage for goods.

A pre-requisite for such a system is, however, that a suitable natural gas engine can be realised.

\subsection{Prime movers for small scale CHP systems}

Several different designs have been proposed for small scale CHP systems, however there is currently only a limited number of units commercially available. The smallest units, such as the WhisperGen micro-CHP system manufactured by WhisperGen Ltd. [2], are mainly based on the Stirling engine, and have electric power output down to around $1 \mathrm{~kW}_{e}$. Although the Stirling engine is capable of electric generation efficiencies of up to 25 per cent, units currently available in this size range typically have lower ratios of electric to heating power generation. Allen et al. [3] stated that there are currently around 1000 micro-CHP units installed in the UK, and that such units are financially viable with payback time of $3 \rightarrow 5$ years.

Internal combustion engines are generally considered unsuitable for micro-scale CHP systems due to factors such as high levels of vibration and noise, higher exhaust gas emissions levels compared to continuous combustion systems, less fuel flexibility, and shorter lifetime. Some examples do, however, exist, such as the $5.5 \mathrm{~kW}_{e}$ Dachs micro-CHP unit manufactured by SenerTec GmbH [4], and such engines have some clear advantages over existing external 
combustion technologies, including high power density, simple control, and high fuel efficiency. In addition, internal combustion engine technology is wellproven and highly developed. With the low price, high availability, and well established distribution networks for natural gas existing in many countries, including the United Kingdom, natural gas internal combustion engines present a potential option for use in a combined heat and power system. If the challenges of noise and vibration can be overcome, for example through acoustic insulation in the mounting of the unit, there is no reason why internal combustion engines should not be feasible for micro-scale CHP units.

Currently, small scale internal combustion engines are mainly used in applications such as small motorbikes and portable electric generators, in which the requirement of a high power to weight ratio is that of highest importance. In the design of a combustion engine there is a clear trade-off between power density and efficiency, and, since the power density requirement is relaxed in stationary applications such as a CHP system, the engine can be optimised for high efficiency and long life. Engine optimisation may include factors such as: the use of a high stroke to bore ratio to reduce in-cylinder heat transfer losses; reducing engine mean piston speed to minimise frictional and gas flow losses; reducing the compression ratio to reduce sealing requirements; lean burn operation to reduce peak incylinder gas temperatures and pressures; and the use of an over-expanded cycle to maximise the mechanical work extracted from the cylinder gases. Since most of these result in a reduction in engine power to weight ratio, they are normally not used in existing small scale internal combustion engines. Hence, there is a significant potential for enhancing engine fuel efficiency if a penalty in engine size and weight can be accepted.

\subsection{The Miller cycle engine}

The Miller cycle was proposed by Miller [5], with the main objective of improving engine efficiency. It is an over-expanded cycle, i.e. a cycle with an expansion ratio higher than its compression ratio. Recently, the Miller cycle has also been proposed as a means of reducing harmful $\mathrm{NO}_{x}$ emissions while maintaining a high engine efficiency, by reducing the engine compression ratio and thereby also peak incylinder gas temperatures and pressures.
A number of reports have described the Miller cycle engine concept and investigated various aspects of its design and operation. Al-Sarkhi et al. [6] and Zhao and Chen [7] presented theoretical investigations into Miller cycle engine performance, studying the influence of the main engine design variables and system irreversibilities. Endo et al. [8] described the design of a commercially available large scale (280$1100 \mathrm{~kW}$ ) gas engine using the Miller cycle principle, claiming a fuel efficiency advantage of more than $5 \%$ over comparable conventional technology. Gheorghiu and Ueberschär [9] studied an overexpanded engine for use in hybrid vehicles and investigated sources of efficiency loss in the conventional implementation of such cycles. Wang and Ruxton [10] and Wang et al. [11] investigated the application of the Miller cycle concept to reduce engine exhaust gas emissions and found that significant $\mathrm{NO}_{x}$ reductions could be achieved, albeit with a penalty in engine fuel consumption.

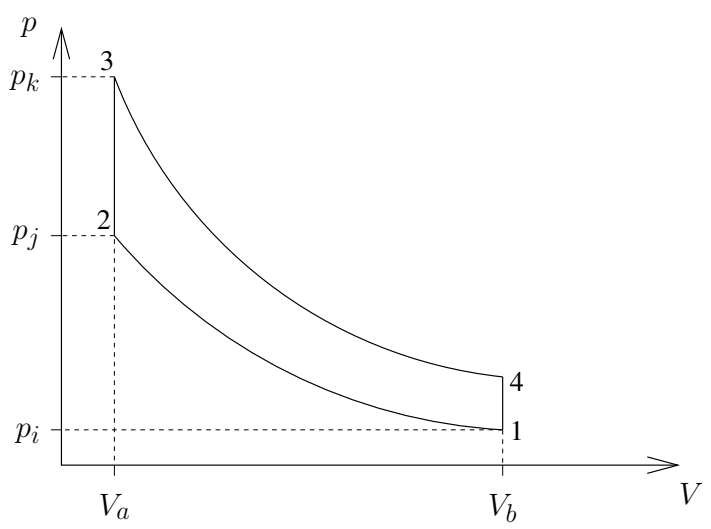

(a) Air-standard Otto cycle.

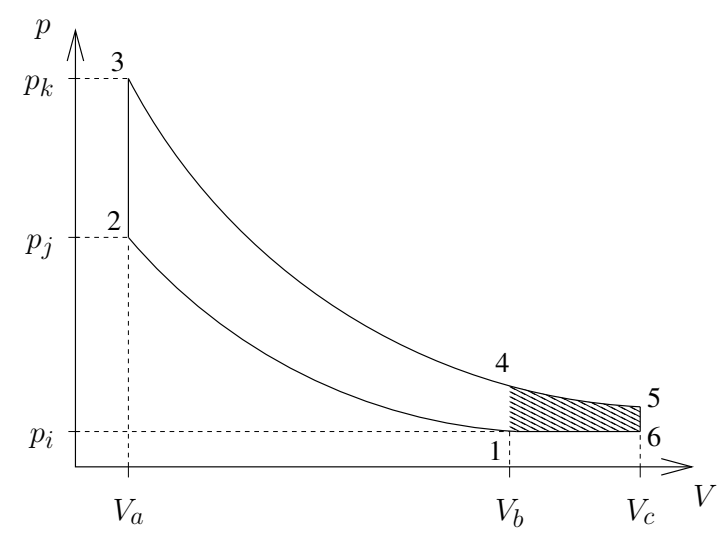

(b) Air-standard Miller cycle.

Fig. 2. Comparison of Otto and Miller air-standard cycles. 
Figure 2 shows the air-standard Otto and Miller cycles, and illustrates the additional work that can be extracted from the Miller cycle (shaded). Heywood [12] showed that significant increases in engine efficiency can be achieved in over-expanded cycles, especially at low compression ratios.

\subsection{Realising the Miller engine}

A number of recent studies have investigated the modification of existing conventional engines for operation on a Miller cycle. This can be achieved by advancing the closing of the inlet valves, thereby limiting the amount of fresh charge that will flow into the cylinder, or by delaying the closing so that a part of the inlet charge is rejected. By lowering the effective compression ratio, the peak in-cylinder gas temperatures and pressures are reduced, which reduces the formation of temperature-dependent emissions such as nitrogen oxides. The expansion ratio is maintained, limiting the fuel efficiency penalty associated with lower compression ratios.

The current work seeks a more fundamental approach to the design of a Miller cycle engine, investigating an engine purposely designed for Miller cycle operation. The study will be based on a standard Otto-cycle spark ignition engine, and a Miller cycle version of the same engine will be designed by increasing the stroke length. This allows a direct comparison of the performance of the two engines to be undertaken, allowing investigations into the potential advantages and disadvantages of the Miller cycle engine.

\section{Thermodynamic analyses}

The basic features of the two engine cycles can be investigated theoretically using cycle analyses. Having an Otto-cycle engine, such as the one illustrated in Figure 2a, the Miller cycle version can be created by increasing the stroke length. The compression is assumed to start at the same point, however the expansion ratio will be increased as can be seen in Figure $2 b$.

The additional expansion work from the cycle can now be estimated. The compression work, $W_{c}$, and the expansion work, $W_{e}$, for the Otto-cycle engine are

$$
\begin{aligned}
& W_{c, \text { otto }}=\int_{V_{1}}^{V_{2}} p \mathrm{~d} V \\
& W_{e, \text { otto }}=\int_{V_{3}}^{V_{4}} p \mathrm{~d} V
\end{aligned}
$$

and for the Miller cycle engine

$$
\begin{aligned}
& W_{c, \text { miller }}=\int_{V_{6}}^{V_{1}} p \mathrm{~d} V+\int_{V_{1}}^{V_{2}} p \mathrm{~d} V \\
& W_{e, \text { miller }}=\int_{V_{3}}^{V_{4}} p \mathrm{~d} V+\int_{V_{4}}^{V_{5}} p \mathrm{~d} V,
\end{aligned}
$$

where $p$ is in-cylinder gas pressure, $V$ is gas volume and the subscripts denote the points on the airstandard cycles shown in Figure 2.

Assuming that the in-cylinder gases follow polytropic compression and expansion state changes, the in-cylinder pressure can be described by

$$
p=p_{0} V_{0}^{n} \frac{1}{V^{n}}
$$

where the subscript 0 denotes starting conditions and $n$ is the polytropic coefficient. Inserting this into the integral and solving, one can derive expressions for the net output work $\left(W_{e}-W_{c}\right)$ for the two cycles as functions of the volumes $V_{a}, V_{b}, V_{c}$ and pressures $p_{i}, p_{j}, p_{k}$.

The compression ratio $r_{c}=V_{b} / V_{a}$ will be equal for the Otto and Miller engines. For the Otto cycle engine, the expansion ratio is equal to the compression ratio, whereas for the Miller cycle engine, one can define a 'Miller cycle ratio', $\varepsilon$, expressing the relative difference between expansion ratio and compression ratio:

$$
\varepsilon=\frac{r_{e}}{r_{c}}=\frac{V_{c} / V_{a}}{V_{b} / V_{a}}=\frac{V_{c}}{V_{b}}
$$

where $r_{e}$ is the Miller cycle expansion ratio.

\subsection{Efficiency advantages from the expanded cycle}

The compression ratio, $r_{c}$, in modern conventional spark ignited engines is typically limited to around 10 due to fuel autoignition limitations. Natural gas and some other alternative fuels, such as ethanol, have higher knock resistance (octane number), and may utilise higher compression ratios. For applications such as the one investigated here, a lower compression ratio is, however, desirable in order to re- 
duce cylinder sealing requirements, frictional losses and engine noise.

The maximum cycle pressure $p_{k}$ will depend on the amount of heat added to the charge in the constant-volume process 2-3. For spark ignition engines, the ratio of end-of-compression pressure $\left(p_{j}\right)$ to peak pressure $\left(p_{k}\right)$ is typically around 3 at full load.

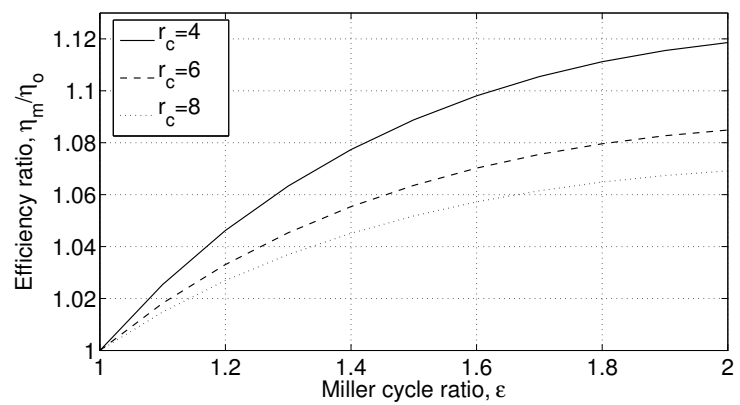

(a) Efficiency advantage of the Miller cycle for varying compression ratios $\left(p_{3} / p_{2}=3\right)$.

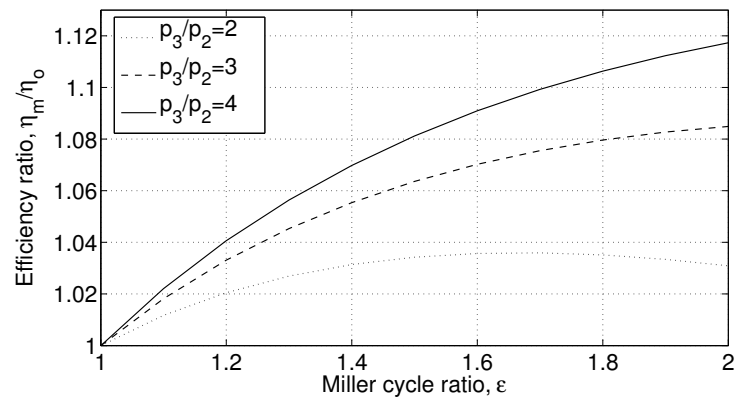

(b) Efficiency advantage of the Miller cycle for varying heat inputs $\left(r_{c}=6\right)$.

Fig. 3. Potential efficiency advantage of the Miller cycle engine.

Figure 3 shows the air-standard efficiency advantage that can be achieved in a Miller cycle engine compared to the equivalent Otto cycle. It is seen that the Miller cycle advantages are larger for engines with low compression ratios, which is consistent with reports from other authors [12]. Furthermore, a significant influence of the heat input is seen, indicating that the Miller cycle is suitable for engines operating at high loads, and possibly more beneficial for fuels with high heat content.

\subsection{Reduction in power output}

The operating speed of an engine is limited by resistance to gas flows and mechanical stress of en- gine components. Engine efficiency is highly influenced by pressure losses over valves, and in an engine such as the one investigated here, a low mean piston speed is desirable in order to minimise such pumping losses. Modifying the engine design to a longer stroke length, as described above, will require a reduced operating speed to maintain a constant mean piston speed, and will therefore lead to a reduction in engine power output.

Mean piston speed, $\bar{S}_{p}$, is defined as

$$
\bar{S}_{p}=2 S N
$$

where $S$ is engine stroke length and $N$ is operating speed. The power output, $P$, from the engine can be expressed as

$$
P=W_{\text {net }} N \frac{1}{n_{R}}=W_{\text {net }} \frac{\bar{S}_{p}}{2 S} \frac{1}{n_{R}}
$$

where $W_{\text {net }}$ is the net work output from the cycle, $N$ is engine operating speed and $n_{R}$ is the number of engine revolutions per power stroke ( 2 for four stroke engines).

It is seen that for a constant work output per cycle and a constant mean piston speed, the power output of the engine scales inversely proportional to stroke length. In the Miller cycle engine, the work output is not constant, but increases with increasing stroke length, and that must be taken into account in this analysis.

The ratio of power outputs becomes

$$
\frac{P_{\text {miller }}}{P_{\text {otto }}}=\frac{W_{\text {net,miller }}}{W_{\text {net,otto }}} \frac{S_{\text {otto }}}{S_{\text {miller }}},
$$

and the ratio of stroke lengths in the Miller and Otto engines can be expressed in terms of compression ratios:

$$
\frac{S_{\text {otto }}}{S_{\text {miller }}}=\frac{r_{c}-1}{r_{e}-1}
$$

Figure 4 shows the theoretical power reduction in the Miller cycle engine due to reduced engine speed. A significant reduction can be seen, as expected. The power reduction is, however, seen not to be largely influenced by the engine compression ratio or heat input.

\section{CFD simulation of Miller cycle engine performance}

In order to investigate the effects of employing the Miller cycle in a real engine, a simulation model 


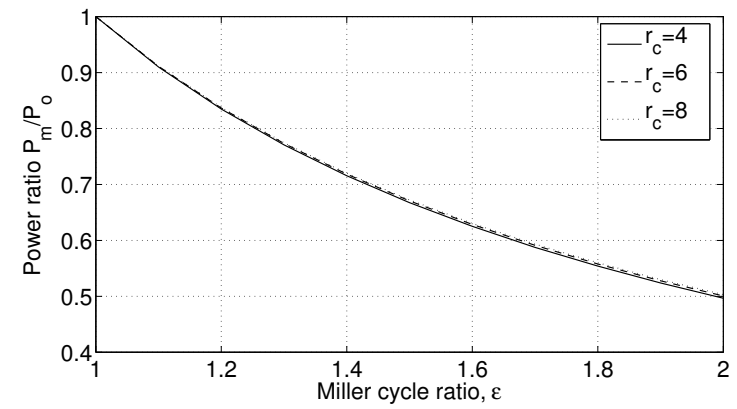

(a) Power reduction in the Miller cycle for varying compression ratios $\left(p_{3} / p_{2}=3\right)$.

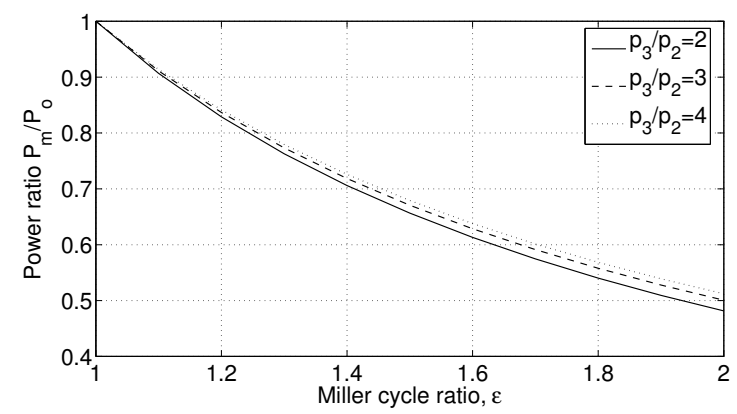

(b) Power reduction in the Miller cycle for varying heat inputs $\left(r_{c}=6\right)$.

Fig. 4. Power reduction in the Miller cycle engine with constant mean piston speed.

was set up using the computational fluid dynamics (CFD) toolkit OpenFOAM [13,14]. A multidimensional simulation model takes into account important factors such as in-cylinder fluid flow and heat transfer, and will give better insight into the potential advantages of the Miller cycle engine than basic cycle analyses do.

The simulations were performed by comparing a standard, Otto-cycle engine with alternative configurations of the same engine with an increased stroke length, as above. The compression ratio is held constant, whereas the expansion ratio increases as the stroke length is increased.

\subsection{Engine configuration}

The modelled engine is a standard spark ignition engine with main engine data as shown in Table 1.

With a brake mean effective pressure (bmep) of $300 \mathrm{kPa}$, such an engine would produce around $1 \mathrm{~kW}$ of output power per cylinder.

\begin{tabular}{|c|c|}
\hline Stroke & $0.060 \mathrm{~m}$ \\
\hline Bore & $0.050 \mathrm{~m}$ \\
\hline Speed & $3000 \mathrm{rpm}$ \\
\hline Aspiration & natural \\
\hline Compression ratio & 6 \\
\hline
\end{tabular}

Table 1

Engine specifications.

\subsection{Simulation setup}

The simulation model had previously been validated against experimental results from an automotive spark ignition engine modified to run on a Miller cycle, presented by Wang and Ruxton [10]. A simplified model of the engine was developed and a mesh of the engine cylinder was created. A simple combustion chamber design with a standard bowlin-piston design with a cone-shaped cylinder head was used. The combustion chamber is assumed symmetric around the cylinder axis, which allows a wedge geometry with cyclic boundary conditions to be used, reducing computational costs. Since the study is parametric in nature and only investigates trends in engine performance, the specific design of the combustion chamber has little influence on the results and these simplifications are acceptable.

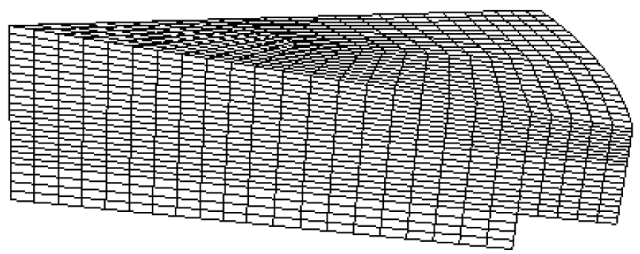

Fig. 5. Computational mesh.

Figure 5 shows the computational mesh used in the simulations. The mesh is a 30 -degree wedge with 10,600 cells, equivalent to more than 120,000 cells for the full cylinder. Mesh sensitivity analyses were performed to ensure the independence of the solution of the mesh density. Combustion was modelled with the Weller combustion model [15], with a centrally located spark plug. Turbulence was modelled with a standard $k-\epsilon$ model with wall functions, and constant wall temperatures were used. Thermodynamic properties are calculated using JANAF thermochemical tables [14]. Swirl was introduced at $\mathrm{BDC}$, and the swirl level at BDC was equal for all designs. The fuel used was methane (the main con- 
stituent of natural gas) at a fuel-air equivalence ratio of 1 .

\subsection{Simulation results}

Simulation cases were set up for the original, Ottocycle engine and for Miller cycle engines with $\epsilon=$ $1.2, \epsilon=1.4$ and $\epsilon=1.6$. The optimum value for ignition timing (that giving the highest efficiency) varies between the designs, among other things due to the differences in engine speed. The optimum spark timing was identified for all designs, and the results presented here are all run under these conditions.

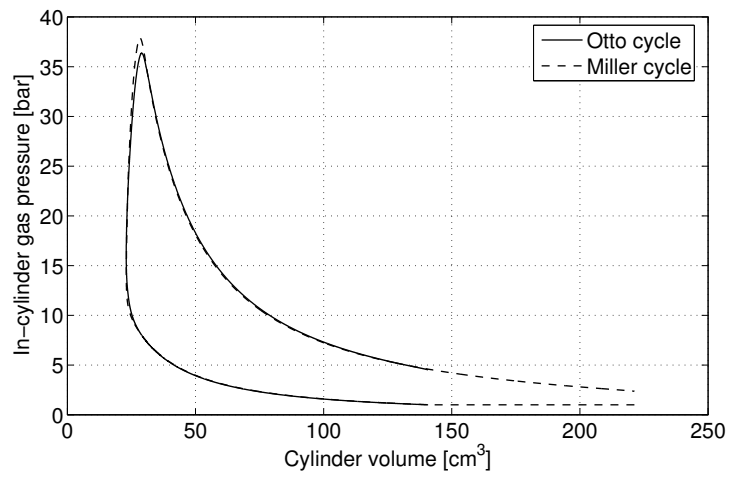

Fig. 6. Pressure-volume plot for the original engine and the Miller cycle engine with $\epsilon=1.6$.

Figure 6 shows the $p$ - $V$ diagrams of the Otto-cycle engine and the Miller cycle engine with $\epsilon=1.6$. It was found that the combustion in the Miller cycle engine benefits from the lower engine speed, and is slightly closer to a constant-volume process. The additional expansion work from the Miller cycle is seen to add significantly to the original (Otto-cycle) net cycle work.

Figure 7 shows the predicted engine performance for the original, Otto-cycle engine $(\epsilon=1)$ and the Miller cycle engine for different Miller cycle ratios. In Figure $7 \mathrm{a}$ it can be seen that the indicated efficiency benefits from the increased expansion ratio, as expected. The efficiency advantage for the Miller cycle engine is higher than that predicted in the cycle analyses. This is due to the fact that the efficiency in a real engine is determined by a number of factors, including heat transfer losses, volume change during combustion, and in-cylinder gas motion, that are not taken into account in the cycle analyses.

The engine indicated power is seen to drop with the increasing expansion ratio, however the reduction is slightly lower than that predicted above. It

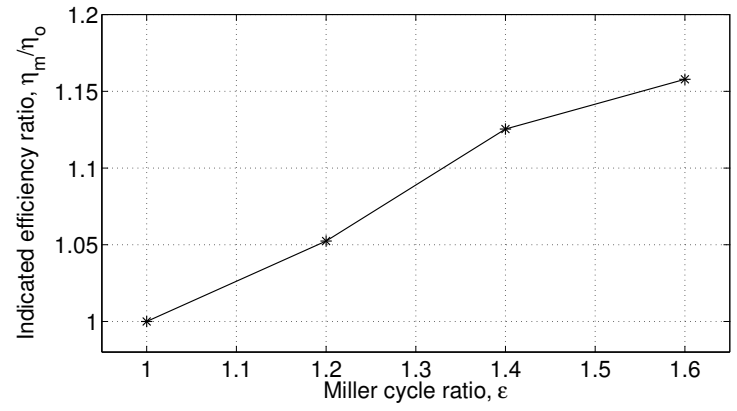

(a) Indicated efficiency advantage of the Miller cycle.

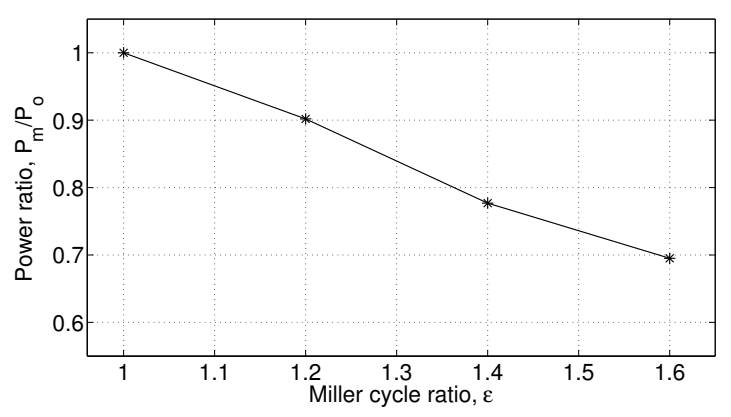

(b) Power reduction in the Miller cycle.

Fig. 7. Predicted Miller cycle performance.

should be noted that in addition to reducing the power output of the engine, modifying a conventional engine to run on the Miller cycle as done here will also increase engine weight. Hence, the engine power to weight ratio will be influenced to a higher degree than the power ratio shown in the figure. Moreover, these analyses are based on indicated (incylinder) values. Differences in the mechanical efficiency of the engine will influence both the fuel efficiency and the output power, and must therefore also be taken into account when evaluating the potential advantages of the Miller cycle concept.

\section{Frictional losses in the Miller cycle engine}

Frictional losses relate the indicated work (the work done on the pistons by the in-cylinder gases) to the actual output work from the engine and are of high importance in internal combustion engines. Engine friction is typically divided into (a) the pumping work required to draw fresh mixture into the cylinders and expel the burnt gases; (b) the work required to drive engine accessories such as water and oil pumps; and (c) the mechanical friction between moving parts in the engine. In small scale engines, typically between 20 per cent (at full load) and 100 
per cent (at idle) of the indicated work is lost to friction.

The increased stroke length and swept volume in the Miller cycle engine will lead to an increase in frictional losses, and, hence, the efficiency advantage found above will be reduced. Of the three sources of friction listed above, the main difference between the two engines will be in the mechanical friction. The power required to drive the valve chain and engine accessories will not differ significantly between the two engines, and there will be no major differences in pumping losses due to the constant mean piston speed.

Piston-cylinder friction typically accounts for around half of the frictional losses in a spark ignition engine [12]. Although piston-cylinder friction will increase with increasing stroke length, it is not likely to scale proportionally to the Miller cycle ratio due to the differences in the in-cylinder pressures during the different parts of the cycle. The overexpanded part of the cycle will have significantly lower in-cylinder gas pressures than the part of the cycle that is 'shared' between the Otto and Miller cycles and friction from piston rings, pistons and bearings will therefore be lower.

Consider an engine in which 20 per cent of the indicated work is lost to friction and assume that half of this is related to friction mechanisms that will be influenced by the Miller cycle ratio. If this friction increase proportionally to $\epsilon$ (a pessimistic assumption), a value of $\epsilon$ of 1.2 will increase the frictional losses by $2 \%$ of the indicated work. Comparing this to the results in Figure 7, it is seen that this is less than half of the predicted efficiency advantage in the Miller cycle engine. The increase in net cycle work in the Miller cycle engine is therefore likely to outweigh the increased frictional losses resulting from the increased stroke length.

\section{Conclusions}

This paper has investigated the potential benefits of applying the Miller cycle concept to a small scale natural gas engine suitable for applications such as domestic combined heat and power systems. It was found that the Miller cycle has potential fuel efficiency advantages compared to a conventional Ottocycle engine, however with the cost of a reduced engine power density. An increase in frictional losses is expected in the Miller cycle engine, but a simple analysis indicated that these will be outweighed by the fuel efficiency benefits of the Miller cycle.

Further work is required to validate the findings presented in this paper, and a more detailed investigation into engine frictional losses, noise and vibration, and system lifetime should be undertaken. The preliminary findings are, however, encouraging, and suggest that significant fuel efficiency improvements can be achieved in small scale internal combustion engine with the use of an over-expanded cycle, provided that a penalty in engine power density can be accepted.

\section{References}

[1] The CarbonNeutral Company, http://www . carbonneutral .org.

[2] WhisperGen Ltd, http://www. whispergen.com.

[3] Allen SR, Hammond GP, McManus MC. Prospects for and barriers to domestic micro-generation: A United Kingdom perspective Applied Energy, 2008:85:528-544.

[4] SenerTec GmbH, http://www.senertec.de.

[5] Miller RH. Supercharging and internal cooling cycle for high output Transactions of ASME 1947:69:453-457.

[6] Al-Sarkhi A, Jaber JO, Probert SD. Efficiency of a Miller engine. Applied Energy 2006:83:343-351.

[7] Zhao Y, Chen J. Performance analysis of an irreversible Miller heat engine and its optimum criteria. Applied Thermal Engineering 2007:27:2051-2058.

[8] Endo H, Tanaka K, Kakuhama Y, Goda Y, Fujiwaka T, Nishigaki M. Development of the lean burn Miller cycle gas engine. In: 5th Int. Symposium on Diagnostics and Modeling of Combustion in Internal Combustion Engines (COMODIA 2001), July 1-4, 2001, Nagoya.

[9] Gheorghiu V, Ueberschär D. Enhancement potential of the thermal conversion efficiency of ICE cycles especially for use in hybrid vehicles. In: 5th Int. Conference on Heat Transfer, Fluid Mechanics and Thermodynamics (HEFEAT2007), Sun City, South Africa, 2007.

[10] Wang Y, Ruxton T. An experimental investigation of NOx emission reduction from automotive engine using the Miller cycle. Proc. ASME 2004 Fall Technical Conference, Long Beach, CA, USA, 2004.

[11] Wang Y, Lin L, Roskilly AP, Zeng S, Huang J, He Y, Huang X, Huang H, Wei H, Li S, Yang J. An analytic study of applying Miller cycle to reduce NOx emission from petrol engine. Applied Thermal Engineering 2007:27:1779-1789.

[12] Heywood, J.B. Internal combustion engine fundamentals McGraw-Hill, Inc., 1988.

[13] OpenFOAM project web site, http://www. OpenFOAM. org, 2007.

[14] Jasak H, Weller HG, Nordin N. In-cylinder CFD simulation using a $\mathrm{C}++$ object-oriented toolkit. SAE Paper 2004-01-0110, Society of Automotive Engineers, 2004.

[15] Weller, HG. The development of a new flame area combustion model using conditional averaging. ThermoFluids Section Report TF 9307, Imperial College of Science, Technology and Medicine, 1993. 